\title{
Magnetic Field-Decay Accelerator Driven by a Relativistic Electron Beam in a Ferromagnetic Waveguide
}

\author{
Han S. Uhm \\ Naval Surface Warfare Center \\ 10901 New Hampshire Ave, White Oak \\ Silver Spring, Maryland 20903-5640
}

Abstract

A novel high-gradient magnetic field-decay accelerator is presented in which the drive-beam leaves behind a high-gradient accelerating field produced by the magnetic field decay. The electromagnetic fields indicate a decaying function of the time. As an example, we obtain the acceleration field for the case of a short and intense drive bunch, which is represented by a parabolic function of time. With appropriate physical parameters, the acceleration gradient of the magnetic field-decay accelerator can be easily more than 150 megavolt/meter.

\section{INTRODUCTION}

In recent years, there has been a strong progress in the high-current electron-beam technology. Electron beams with an energy of $10 \mathrm{MeV}$ and a current of $10 \mathrm{kA}$ are easily available in the present technology. In addition, a tremendous improvement has been made in the effective control of these electron beams, including the focus, modulation, and a timely termination of the beam current. Thus, the electron beam itself is used as a drive current in accelerators. In this presentation, we study the acceleration mechanism based on the magnetic field decay. ${ }^{1}$ The magnetic field energy is stored in a high-permeability material by a continuous beam current. If the current drops abruptly, the magnetic field stored in the material decays, thereby generating an acceleration electric field. Physics of the accelerating field arising from the finite conductivity waveguide is very similar to that of the longitudinal resistive wall instability ${ }^{2,3}$ which has been investigated for application to circular accelerators. In this presentation, a theory of the magnetic field diffusion is developed, in order to estimate the acceleration gradient whenever the sustaining beam current drops. As an example, we obtain the acceleration field for the case of a short and intense drive bunch, which is represented by a parabolic function of time. The acceleration field is given by $E_{m}=$ $150 \mathrm{MV} / \mathrm{m}$, for the waveguide hole radius of $R_{1}=0.5 \mathrm{~cm}$, the saturation magnetic field $B_{s}=15 \mathrm{kG}$, and $\Delta t=10^{-10}$ second. Assuming that the parameter $\mu \sigma=80$ siemens $/ \mathrm{m}$,

This work was supported by the Independent Research Fund at the Naval Surface Warfare Center. the required drive current for the saturation field is given by $\mu I_{m}=660 \mathrm{kA}$, which is equivalent to $I_{m}=660 \mathrm{~A}$ for $\mu=$ 1000. The total charge of this drive beam pulse is $Q=85$ nanocoulomb for $I_{m}=660 \mathrm{~A}$.

\section{EVALUATION OF ACCELERATING FIELD}

The theoretical model is based on the induced electric field due to decay of the field energy stored in an energy storage device. We assume that an electron beam with current $I(t)$ propagates through a hole with radius of $R_{1}$ in the field-energy storage with radius of $R_{2}$. If the current drops abruptly, the magnetic field stored in the material decays, thereby generating an acceleration electric field

$$
\begin{aligned}
E_{z}\left(R_{1}, t\right)= & -\frac{2 \mu}{c^{2}} \int_{0}^{\infty} \frac{d k}{k}\left[J_{0}\left(k R_{2}\right)-J_{0}\left(k R_{1}\right)\right]^{2} \\
& \cdot \int_{-\infty}^{t} d t^{\prime}\left(\frac{d I}{d t^{\prime}}\right) \frac{\partial}{\partial t^{\prime}} q_{k}\left(t-t^{\prime}\right),
\end{aligned}
$$

where the time function $\mathrm{q}_{\mathrm{k}}(\mathrm{x})$ is defined by

$$
q_{k}\left(t-t^{\prime}\right)=\exp \left[-\frac{c^{2} k^{2}}{4 \pi \sigma \mu}\left(t-t^{\prime}\right)\right]
$$

and $\sigma$ and $\mu$ are the conductivity and magnetic permeability of the energy storage material. Substituting Eq. (2) into Eq. (1), we show that the acceleration electric field $E_{z}$ is expressed as

$$
\begin{aligned}
E_{\mathrm{z}}(t) & =-\frac{1}{2 \pi \sigma} \int_{-\infty}^{t} d t^{\prime}\left(\frac{d I}{d t^{\prime}}\right) \int_{0}^{-} d k k\left[J_{0}\left(k R_{2}\right)\right. \\
& \left.-J_{0}\left(k R_{1}\right)\right]^{2} \exp \left[-\frac{c^{2}\left(t-t^{\prime}\right)}{4 \pi \sigma \mu} k^{2}\right] .
\end{aligned}
$$

For convenience in the subsequent analysis, we define the normalized times $\zeta$ and $\zeta^{\prime}$ by

$$
\zeta=\frac{c^{2} t}{2 \pi \sigma \mu R_{1}^{2}}, \quad \zeta^{\prime}-\frac{c^{2} t^{\prime}}{2 \pi \sigma \mu R_{1}^{2}}
$$


Making use of Eq. (4), Eq. (3) is rewritten as

$$
\begin{gathered}
E_{z}(t)=-\frac{1}{2 \pi \sigma R_{1}^{2}} \int_{-\infty}^{t} d t^{\prime}\left(\frac{d l}{d t^{\prime}}\right) \int_{0}^{\infty} d x x\left[J_{0}\left(x R_{2} / R_{1}\right)\right. \\
\left.-J_{0}(x)\right]^{2} \exp \left[-\frac{\left(\zeta-\zeta^{\prime}\right)}{2} x^{2}\right] .
\end{gathered}
$$

The integration over the variable $\mathrm{x}$ is carried out by making use of the integral ${ }^{4}$

$$
\begin{gathered}
\int_{a}^{\infty} x d x \exp \left(-\rho^{2} x^{2}\right) J_{p}(\alpha x) J_{p}(\beta x) \\
=\frac{1}{2 \rho^{2}} \exp \left(-\frac{\alpha^{2}+\beta^{2}}{4 \rho^{2}}\right) I_{p}\left(\frac{\alpha \beta}{2 \rho^{2}}\right)
\end{gathered}
$$

of the Bessel functions. The accelerating field in Eq. (5) is expressed as

$$
\begin{gathered}
E_{z}(t)=-\frac{1}{2 \pi \sigma R_{1}^{2}} \int_{-\infty}^{t} d t^{\prime}\left(\frac{d l}{d t^{\prime}}\right) \frac{1}{\delta \zeta}\left[\exp \left(-\frac{1}{\delta \zeta}\right) I_{0}\left(\frac{1}{\delta \zeta}\right)\right. \\
+\exp \left(-\frac{1}{\delta \zeta} \frac{R_{2}^{2}}{R_{1}^{2}}\right) I_{0}\left(\frac{1}{\delta \zeta} \frac{R_{2}^{2}}{R_{1}^{2}}\right) \\
\left.-2 \exp \left(-\frac{1+R_{2}^{2} / R_{1}^{2}}{2 \delta \zeta}\right) I_{0}\left(\frac{1}{\delta \zeta} \frac{R_{2}}{R_{1}}\right)\right],
\end{gathered}
$$

where $\delta \zeta=\zeta-\zeta^{\prime}$.

The contribution of the second and third terms in the square bracket in the right-hand side of Eq. (7) is less than 20 percent if the parameter $R_{2} / R_{1}$ is larger than five in practical applications. Thus, we neglect these terms and Eq. (7) is simplified to

$$
E_{z}(t)--\frac{1}{2 \pi \sigma R_{1}^{2}} \int_{-\infty}^{t} d t\left(\frac{d I}{d t^{\prime}}\right) \frac{1}{\delta \zeta}\left[\exp \left(-\frac{1}{\delta \zeta}\right) I_{0}\left(\frac{1}{\delta \zeta}\right)\right] .
$$

Recognizing the approximation

$$
\frac{1}{x} \exp \left(-\frac{1}{x}\right) I_{0}\left(\frac{1}{x}\right)-\frac{1}{\sqrt{2 \pi x}}
$$

for the variable $\mathrm{x}$ satisfying $\mathrm{x}<10$, we can further simplify the acceleration field in Eq. (8) by

$$
E_{z}(t)--\frac{1}{(2 \pi)^{3 / 2} a R_{1}^{2}} \int_{-\infty}^{\zeta} \frac{d \zeta^{\prime}}{\sqrt{\zeta-\zeta^{\prime}}}\left(\frac{d l}{d \zeta^{\prime}}\right)
$$

which is one of the main result in this section and can be used to calculate the accelerating field for a broad range of system parameters. Note that the drive current $I(t)$ in Eq. (10) is not specified yet. I remind the reader that Eq. (10) is valid for $\zeta$ $-\zeta^{\prime}<10$. This requires that the observation time $\zeta$ must be close to the time $\zeta^{\prime}$, at which the maximum current-change occurs. As will be seen later, the peak acceleration field occurs right after the maximum current decrease. Therefore, the restriction $\zeta-\zeta^{\prime}<10$ is well justified. Similarly, the azimuthal magnetic field at $r=R_{1}$ is obtained from $B_{\theta}\left(R_{1}, t\right)$ $=-(\partial / \partial r) A_{z}(r, t)$ and given by

$$
B_{\theta}\left(R_{1}, \zeta\right)=\frac{\mu}{2 \sqrt{2 \pi} R_{1} c} \int_{-\infty}^{\zeta} \frac{d \zeta^{\prime}}{\sqrt{\zeta-\zeta^{\prime}}} I\left(\zeta^{\prime}\right),
$$

which will be used to estimate the magnetic field at $r=R_{1}$ only for a fast-changing drive current.

As an example, we consider the case when the drive electron beam has a very short pulse with the pulse profile defined by

$$
I(t)=I_{m}\left(1-\frac{t^{2}}{\Delta t^{2}}\right) U\left(\Delta t^{2}-t^{2}\right),
$$

where $\Delta t$ is a constant related to the heam pulse and $U(x)$ is the Heaviside step function. Substituting Eq. (12) into Eq. (11) and carrying out the integration over the time $t^{\prime}$, we obtain the acceleration field

$$
E_{z}(t)=\frac{2 I_{m}}{3 \pi c R_{1}} \sqrt{\frac{\mu}{\sigma \Delta t}} q\left(\frac{t}{\Delta t}\right),
$$

where the function $q(u)$ is defined by

$$
q(u)=\left\{\begin{array}{l}
\sqrt{u+1}(2 u-1),-1<u<1, \\
\sqrt{u+1}(2 u-1)-\sqrt{u-1}(2 u+1), u>1 .
\end{array}\right.
$$

Shown in Fig. 1 is plot of the function $q(u)$ versus the normalized time $u$ obtained from Eq. (14). Value of the function $q$ at early stage of the drive-beam pulse is negative, indicating that the drive beam transfers its kinetic energy into the field energy in the energy storage device. This large negative value of the function $q$ inside the drive pulse decelerates the beam itself quickly. In this regard, the pulse profile in Eq. (12) is not appropriate for efficient acceleration of the witness beam. The negative value of $q(u)$ in the pulse must be minimized by a gradual increase of the drive-beam current. However, this simple profile is good for the feasibility study, which is the purpose of this article. As shown in Fig. 1, the function $q$ increases from its minimum as the normalized time $u$ increases from $u=-0.5$ to 1 . By 
self-evolution, this portion of the drive pulse will stiffen the termination slope of the current, eventually increasing the peak value of the function $q$. The peak value of the function $q(u)$ occurs at $\mathbf{u}=1$, where the drive pulse ends. Electrons trailing the drive beam get their maximum energy gain. In reality, the peak value of the function $q$ measured at the axis occurs little later than $u=1$ because there is a traveling time of the acceleration field from $r=R_{1}$ to $r=0$. Eq. (14) (or Fig. 1) exhibits that there is no negative value of $q(u)$ outside the drive beam pulse $(u>1)$. In this regard, this system is not useful for acceleration of positrons. The positive peak value is about $q(u=1)=1.5$. Therefore, the maximum acceleration field is given by

$$
E_{m}-\frac{I_{m}}{\pi c R_{1}} \sqrt{\frac{\mu}{\sigma \Delta t}}
$$

from Eq. (13).

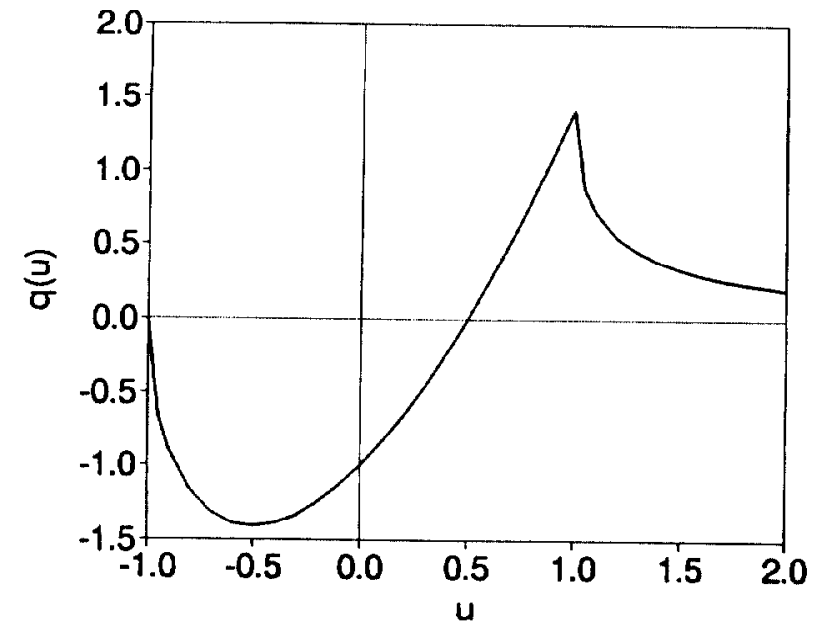

Fig. 1. Plot of the function $q(u)$ versus the normalized time $u$ obtained from Eq. (14) for the drive current profile in Eq. (12).

It is important to determine the magnetic field at $r=$ $R_{1}$ because the field there must be less than the saturation value $B_{\mathrm{s}}$. Substituting Eq. (12) into Eq. (11) and carrying out a straightforward calculation, we obtain

$$
B_{\theta}\left(R_{1}, t\right)-\frac{2 I_{m} \Delta t}{15 \pi R_{1}^{2}} \sqrt{\frac{\mu}{\sigma \Delta t}} g\left(\frac{t}{\Delta t}\right),
$$

where the function $g(u)$ is defined by

$$
g(u)=\sqrt{u+1}\left(3+u-2 u^{2}\right) .
$$

The magnetic field increases from zero and reaches its peak value of 3.67 at $u=0.5$ and decreases as the time progresses from the head of the beam to infinity. Making use of this peak value, we can approximately relate the drive current to the saturation field by

$$
B_{s}-\frac{I_{m} \Delta t}{2 \pi R_{1}^{2}} \sqrt{\frac{\mu}{\sigma \Delta t}} .
$$

Eliminating the beam current in favor of the saturation field $\mathrm{B}_{\mathrm{s}}$, the maximum acceleration field is expressed as

$$
E_{m}-\frac{2 R_{1}}{c \Delta t} B_{s}
$$

which is remarkably proportional to the hole radius $R_{1}$. For specified saturation field and pulse length, the acceleration field increases linearly as the hole radius increases.

As an example, we consider the case of $R_{1}=0.5$ $\mathrm{cm}$, the saturation magnetic field $B_{s}=15 \mathrm{kG}$, and $\Delta t=$ $10^{-10}$ second. The acceleration field for these parameters is given by $\mathrm{E}_{\mathrm{m}}=150 \mathrm{MV} / \mathrm{m}$ obtained from Eq.(19). Assuming that the parameter $\mu \sigma=80$ siemens $/ \mathrm{m}$, the required drive current for the saturation field is given by $\mu \mathrm{I}_{\mathrm{m}}=660 \mathrm{kA}$, which is equivalent to $I_{m}=660 \mathrm{~A}$ for $\mu=1000$. The total charge of this drive beam pulse is $Q=85$ nanocoulomb for $I_{m}=660$ A. Therefore, the acceleration field per unit charge for these parameters is given by $E_{m} / Q=1.8 \mathrm{MV} / \mathrm{m} / \mathrm{nC}$, which is similar to the result obtained from the wakefield accelerator study for similar physical parameters.

A high-current relativistic electron beam with relatively low energy propagates through a hole bored along the field storage device. Obviously, there should be a physical mechanism to maintain an equilibrium condition of the beam, The axial magnetic field is an excellent means to hold the beam electrons together. However, in this case, the magnetic field may not penetrate well through the highpermeability material. We thus propose to use the ionfocused-regime (IFR) propagation ${ }^{5}$ of the electron beam. When a relativistic electron beam propagates through a preionized channel, channel electrons are expelled by the electrostatic force generated by the head of the beam, leaving an ion channel behind. This ion channel partially neutralizes the space charge field of the electron beam, thereby permitting a focused beam. The IFR propagation of a relativistic electron beam has been well demonstrated.

\section{REFERENCES}

[1] H. S. Uhm, Proc. of 1991 IEEE Particle Accelerator Conference May 6-9, 1991, San Francisco, Cal. Vol. IV, 2566 (1991).

[2] V. K. Neil and A. M. Sessler, Rev. Sci. Instrum. 36, 429 (1965).

[3] H. S. Uhm, Phys. Fluids 25, 690 (1982).

[4] I. S. Gradshteyn and I. M. Ryzhik, Table of Integrals, Series, and Products, (Academic Press, New York 1980) Chap. 6.

[5] R. Smith, R. F. Schneider, M. J. Rhee, H. S. Uhm and W. Namkung, J. Appl. Phys. 60, 4119 (1986). 